\title{
ALTERAÇÕES NA MATÉRIA ORGÂNICA E NA BIOMASSA MICROBIANA EM SOLO DE MATA NATURAL SUBMETIDO A DIFERENTES MANEJOS ${ }^{1}$
}

\author{
MILTON MARCHIORI JÚNIOR² e WANDERLEY JOSÉ DE MELO 3
}

\begin{abstract}
RESUMO - Avaliou-se, em um Latossolo Roxo, o efeito de diferentes formas de manejo do solo sobre a matéria orgânica do solo e na biomassa microbiana. Os tratamentos usados foram: mata natural; mata natural até 1976 e café até 1994 (amostragem na projeção da copa e na entrelinha); mata natural até 1976, café até 1991 e milho até 1994; mata natural até 1940, café até 1960, citros até 1978, e cana-de-açúcar até 1994 (amostragem na linha e na entrelinha). A mata natural apresentou os maiores valores de C orgânico no solo e na fração humina e os menores valores foram obtidos nas áreas com cana-de-açúcar, que apresentaram os maiores valores de $\mathrm{C}$ microbiano em relação à mata natural. $\mathrm{O}$ uso agrícola do solo aumentou a porcentagem de $\mathrm{C}$ orgânico na forma de ácidos húmicos e fúlvicos, em relação à mata natural. Em geral, o solo apresentou mais de 74\% do C orgânico na forma de húmus residual.
\end{abstract}

Termos para indexação: manejo do solo, uso do solo, ácido húmico, humina, ácido fúlvico, carbono orgânico, carbono microbiano, compostos orgânicos.

CHANGES IN ORGANIC MATTER AND IN MICROBIAL BIOMASS OF A NATURAL FOREST SOIL UNDER DIFFERENT MANAGEMENT

\begin{abstract}
The effect of soil management on forms of carbon and microbial biomass was studied in a Typic Euthortox soil. The treatments tested were: natural forest; natural forest till 1976 and coffee till 1994 (sampling on the shoot projection and between lines ); natural forest till 1976, coffee till 1991 and corn till 1994; natural forest till 1940, coffee till 1960, citrus till 1978 and sugar cane till 1994 (sampling on the planting line and between lines ). The treatment with sugar cane presented the largest values of microbial carbon in relation to the natural forest. The agricultural management of soil increased the percentage of organic carbon in humic and fulvic acids forms. The soil presented more than $74 \%$ of organic carbon in the form of residual humus.
\end{abstract}

Index terms: soil management, land use, humic acids, fulvic acids, humin, organic carbon, microbial carbon, organic compounds.

\section{INTRODUÇÃO}

O uso do solo na agricultura, após retirada da vegetação natural, tem freqüentemente mostrado alterações nas propriedades químicas e biológicas do solo, as quais são dependentes das condições do solo, do clima, do tipo de cultura e das práticas culturais adotadas. A interação destas condições estabelece uma nova condição de equilíbrio no sistema solo.

\footnotetext{
${ }^{1}$ Aceito para publicação em 7 de julho de 1999 Apresentado no XII International Symposium on Environmental Biogeochemistry, Rio de Janeiro, RJ.

${ }^{2}$ Eng. Agrôn., M.Sc., UNESP-FCAV, Av. Pintos, 1341, CEP 14870-000 Jaboticabal, SP. Bolsista da FAPESP E-mail: mmarchi@fcav.unesp.br

${ }^{3}$ Eng. Agrôn., Dr., Prof. Titular, Dep. de Tecnologia, UNESP-FCAV, Rod. Carlos Tonanni, km 5, CEP 14870-000 Jaboticabal, SP.
}

Segundo Powlson et al. (1987), um fator que responde mais rapidamente às mudanças de uso do solo, que representa uma alteração no aporte anual de material orgânico, é o parâmetro biomassa microbiana. A biomassa microbiana foi definida por Jenkinson \& Ladd (1981), como a parte viva da matéria orgânica do solo, excluindo raízes e animais maiores que $5 \times 10^{-15} \mathrm{~m}^{2}$. Esta constitui um meio de transformação para todos os materiais orgânicos do solo, e atua como reservatório de nutrientes disponíveis às plantas.

Modificações mensuráveis na biomassa microbiana do solo têm sido observadas em razão das práticas de preparo do solo, do manejo de plantas, e da adubação.

A derrubada da mata tropical, seguida de queima da vegetação, provoca uma queda inicial com posterior elevação do nível de biomassa microbiana do 
solo, determinando, ainda, uma nova distribuição ao longo do perfil (Cerri et al., 1985; Geraldes et al., 1995).

Por outro lado, Klemedtsson et al. (1987) não detectaram mudanças significativas na biomassa microbiana, em solos utilizados para cultivo da cevada. Marchiori Júnior (1998), analisando o C microbiano, encontrou teores semelhantes entre pastagens com 20 ou 25 anos e mata natural. No entanto, este mesmo autor encontrou, em áreas cultivadas com algodoeiro por 10 anos, reduções superiores a $60 \%$ no $\mathrm{C}$ microbiano, em relação à mata natural.

Portanto, a matéria orgânica e a biomassa microbiana dos solos podem ser alteradas com maior ou menor intensidade, dependendo do sistema agrícola instalado.

O objetivo deste trabalho foi analisar as formas do carbono da matéria orgânica e o carbono da biomassa microbiana de um Latossolo Roxo sob mata natural, submetido a diferentes formas de manejo agrícola.

\section{MATERIAL E MÉTODOS}

As amostras de terra foram coletadas em unidade de solo pertencente ao grande grupo Latossolo Roxo, textura argilosa. A análise granulométrica na profundidade de $0-20 \mathrm{~cm}$ mostrou os seguintes resultados: argila $(580 \mathrm{~g} / \mathrm{kg})$, limo $(160 \mathrm{~g} / \mathrm{kg})$, areia fina $(200 \mathrm{~g} / \mathrm{kg})$, e areia grossa $(60 \mathrm{~g} / \mathrm{kg})$. As áreas estudadas apresentaram, em média, as seguintes características químicas em amostras de solo coletadas na camada de 0-20 cm, em 1993, antes da amostragem para as análises do trabalho: $\mathrm{M} . \mathrm{O} .=36 \mathrm{~g} / \mathrm{kg} ; \mathrm{pH}\left(\mathrm{em} \mathrm{CaCl}_{2}\right)=4,7 ; \mathrm{Al}+\mathrm{H}=64 \mathrm{mmol}_{\mathrm{d}} \mathrm{dm}^{3}$. $\mathrm{Ca}=20 \mathrm{mmol}_{\mathrm{c}} / \mathrm{dm}^{3} ; \mathrm{Mg}=6 \mathrm{mmol}_{\mathrm{c}} / \mathrm{dm}^{3} ; \mathrm{K}=3,1 \mathrm{mmol}_{\mathrm{d}} / \mathrm{dm}^{3}$; $\mathrm{P}($ resina $)=17 \mathrm{mg} / \mathrm{dm}^{3}$, de acordo com o Instituto Agronômico (1996)

As diferentes formas de manejo do solo constituíramse de quatro áreas, descritas a seguir: a primeira área, com mata natural (condição natural de cobertura do solo); na segunda área, a mata natural foi retirada em 1940, e, depois de sucessivas queimadas, foi instalada a cultura do cafeeiro até 1960, quando este foi substituído pela cultura do citros, explorada economicamente até 1978 , e então, foi introduzida a cultura da cana-de-açúcar, que permaneceu até 1994 (totalizando 54 anos de uso agrícola); na terceira área, a mata natural foi retirada em 1976, sendo em 1977 formado cafezal, o qual foi explorado até 1994 (totalizando 18 anos de uso agrícola). Desta terceira área, uma quarta foi obtida, quando, em 1991, metade da área foi transformada em cultivo de milho até 1994
Para a análise estatística, fez-se uso de um delineamento experimental inteiramente casualizado. Para efeito da verificação de possíveis diferenças entre os usos agrícolas do solo, os dados foram analisados em esquema de parcelas subdivididas: os tratamentos principais foram constituídos por seis manejos do solo, e o tratamento secundário, por duas profundidades de amostragens (PimentelGomes, 1966).

Os tratamentos principais do esquema estatístico foram definidos pelas quatro áreas (já descritas) e duas posições de amostragens nas culturas de cana-de-açúcar e cafeeiro (linha e entrelinha), num total de seis tratamentos. Cada parcela era de1 ha.

Em resumo, os tratamentos ficaram definidos da seguinte maneira: 1- mata natural (MN); 2- mata natural até 1976 e café até 1994, amostragem na projeção da copa (PC); e 3- mata natural até 1976 e café até 1994, amostragem na entrelinha (EL); 4- mata natural até 1976, café até 1991 e milho até 1994 (MI); 5-mata natural até 1940, café até 1960, citros até 1978 e cana-de-açúcar até 1994, amostragem na linha (CL); e 6- mata natural até 1940, café até 1960 , citros até 1978 e cana-de-açúcar até 1994, amostragem na entrelinha (CE).

Utilizaram-se três repetições para cada tratamento e foram coletadas 20 amostras simples nas profundidades de 0-10 e 10-20 cm. Estas foram reunidas por profundidade, formando uma amostra composta por parcela. Portanto, três amostras compostas para cada tratamento.

$\mathrm{Na}$ área com mata, as amostras simples foram obtidas por caminhamento em ziguezague dentro das áreas úteis das parcelas. O mesmo procedimento foi adotado nas parcelas cultivadas com milho, uma vez que as amostras foram obtidas após preparo do solo.

As adubações e calagens foram feitas segundo recomendações oficiais para o Estado de São Paulo.

Nas amostras, após os devidos preparos no laboratório, procedeu-se à determinação do C orgânico (Dabin, 1976), determinação do C microbiano pelo método de fumigação-incubação (Jenkinson \& Powlson, 1976) e extração e fracionamento da matéria orgânica do solo (Duarte, 1994).

\section{RESULTADOS E DISCUSSÃO}

Os resultados apresentados na Tabela 1 indicam que os maiores valores de $\mathrm{C}$ orgânico no solo e na fração humina foram encontrados no tratamento com mata natural, e os menores, no tratamento com canade-açúcar, nas duas profundidades de amostragem. Os teores de C orgânico das frações ácido fúlvico e 
ácido húmico não foram afetados significativamente pelos usos agrícolas do solo. No entanto, ao analisar a porcentagem do $\mathrm{C}$ orgânico do solo presente na forma de ácidos fúlvicos e ácidos húmicos, observa-se que aumentou com os usos agrícolas do solo em relação à mata natural, principalmente na profundidade de $0-10 \mathrm{~cm}$ (Tabela 2). Este fato teve reflexo no teor de matérias húmicas no solo (ácidos fúlvicos + ácidos húmicos), que com os usos agrícolas do solo houve uma tendência de aumentar a porcentagem de $\mathrm{C}$ orgânico na forma de matérias húmicas em relação à mata natural, nas duas profundidades (Tabela 2). Estes valores de matérias húmicas são menores do que os obtidos por Andreaux \& Becerra

TABELA 1. Teor de C orgânico (g/kg) no solo e na fração humina, em amostras de Latossolo Roxo sob diferentes usos do solo, em duas camadas'.

\begin{tabular}{llllll}
\hline Uso do solo $^{2}$ & \multicolumn{2}{c}{$0-10 \mathrm{~cm}$} & & \multicolumn{2}{c}{$10-20 \mathrm{~cm}$} \\
\cline { 2 - 3 } \cline { 5 - 6 } & Solo & Humina & & Solo & Humina \\
\hline MN & $37,2 \mathrm{Aa}$ & $32,8 \mathrm{Aa}$ & & $26,8 \mathrm{Ab}$ & $22,9 \mathrm{Ab}$ \\
MI & $23,3 \mathrm{BCa}$ & $18,2 \mathrm{BCa}$ & & $22,2 \mathrm{BCa}$ & $17,0 \mathrm{ABCa}$ \\
$\mathrm{CL}$ & $18,9 \mathrm{Ca}$ & $14,6 \mathrm{Ca}$ & & $20,5 \mathrm{BCa}$ & $16,6 \mathrm{BCa}$ \\
$\mathrm{CE}$ & $21,8 \mathrm{BCa}$ & $16,3 \mathrm{BCa}$ & & $17,2 \mathrm{Cb}$ & $13,1 \mathrm{Ca}$ \\
PC & $24,9 \mathrm{Ba}$ & $19,9 \mathrm{BCa}$ & & $23,7 \mathrm{ABa}$ & $18,8 \mathrm{BCa}$ \\
EL & $25,3 \mathrm{Ba}$ & $20,6 \mathrm{Ba}$ & & $24,7 \mathrm{ABa}$ & $20,0 \mathrm{ABa}$ \\
\hline
\end{tabular}

${ }^{1}$ Médias seguidas da mesma letra, maiúscula nas colunas e minúscula nas linhas, não diferiram significativamente pelo teste de Tukey a $5 \%$ de probabilidade; coeficiente de variação para tratamentos: $($ solo $=10,5 \%$ e humina $=12,0 \%)$; coefíciente de variação para profundidade $($ solo $=9,1 \%$ e humina $=12,4 \%$ )

2 MN: mata natural; MI: cultivo de milho; CL: cultivo da cana, amostragem na linha; CE: cultivo da cana, amostragem na entrelinha; PC: cultivo do cafeeiro, amostragem na projeção da copa; EL: cultivo do cafeeiro, amostragem na entrelinha

TABELA 2. Distribuição porcentual do C orgânico nas frações da matéria orgânica de um Latossolo Roxo submetido a diferentes usos agrícola, em duas camadas ${ }^{1}$.

\begin{tabular}{|c|c|c|c|c|}
\hline \multirow[t]{2}{*}{ Uso do solo $^{2}$} & \multicolumn{4}{|c|}{ Fração da matéria orgânica ${ }^{3}$} \\
\hline & $(\mathrm{AF} / \mathrm{T}) \times 100$ & $(\mathrm{AH} / \mathrm{T}) \times 100$ & $(\mathrm{MH} / \mathrm{T}) \times 100$ & $(\mathrm{H} / \mathrm{T}) \times 100$ \\
\hline & \multicolumn{4}{|c|}{$0-10 \mathrm{~cm}$} \\
\hline MN & $5,5 \mathrm{Ba}$ & $6,3 \mathrm{Ba}$ & $11,8 \mathrm{Ba}$ & $88,2 \mathrm{Aa}$ \\
\hline MI & $8,4 \mathrm{Aa}$ & $13,8 \mathrm{Aa}$ & $22,2 \mathrm{Aa}$ & $77,9 \mathrm{Ba}$ \\
\hline CL & 7,8Aa & $15,2 \mathrm{Aa}$ & $23,0 \mathrm{Aa}$ & $77,1 \mathrm{ABa}$ \\
\hline $\mathrm{CE}$ & $6,9 \mathrm{Aa}$ & $14,2 \mathrm{Aa}$ & $21,1 \mathrm{Aa}$ & $74,9 \mathrm{Ba}$ \\
\hline $\mathrm{PC}$ & $7,4 \mathrm{Aa}$ & $12,6 \mathrm{Aa}$ & $20,0 \mathrm{Aa}$ & $79,9 \mathrm{ABa}$ \\
\hline \multirow[t]{2}{*}{ EL } & 7,3Aa & $12,0 \mathrm{Aa}$ & $19,3 \mathrm{Aa}$ & $81,2 \mathrm{ABa}$ \\
\hline & \multicolumn{4}{|c|}{$10-20 \mathrm{~cm}$} \\
\hline MN & $6,5 \mathrm{Aa}$ & $8,0 \mathrm{Ba}$ & $14,5 \mathrm{Ba}$ & $85,5 \mathrm{Aa}$ \\
\hline MI & $10,1 \mathrm{Aa}$ & $13,5 \mathrm{Aa}$ & $23,6 \mathrm{Aa}$ & $76,6 \mathrm{Ba}$ \\
\hline CL & $5,1 \mathrm{Aa}$ & $13,7 \mathrm{Aa}$ & $18,8 \mathrm{Aa}$ & $81,2 \mathrm{ABa}$ \\
\hline $\mathrm{CE}$ & $6,1 \mathrm{Aa}$ & $17,8 \mathrm{Aa}$ & 23,9Aa & $76,1 \mathrm{Ba}$ \\
\hline PC & 7,0Aa & $13,6 \mathrm{Aa}$ & $20,6 \mathrm{Aa}$ & $79,4 \mathrm{ABa}$ \\
\hline EL & $5,8 \mathrm{Aa}$ & $13,3 \mathrm{Aa}$ & $19,1 \mathrm{Aa}$ & $81,2 \mathrm{ABa}$ \\
\hline
\end{tabular}

1 Médias seguidas de mesma letra, maiúscula nas colunas e minúscula nas linhas, não diferiram significativamente pelo teste de Tukey a $5 \%$ : coeficiente de variação para tratamentos $(\mathrm{AF} / \mathrm{T}) \times 100=20,2 \% ;(\mathrm{AH} / \mathrm{T}) \times 100=15,8 \% ;(\mathrm{MH} / \mathrm{T}) \times 100=16,1 \% \mathrm{e}(\mathrm{H} / \mathrm{T}) \times 100=5,3 \%$, coeficiente de variação para profundidade: $(\mathrm{AF} / \mathrm{T}) \times 100=25,9 \% ;(\mathrm{AH} / \mathrm{T}) \times 100=15,5 \% ;(\mathrm{MH} / \mathrm{T}) \times 100=20,0 \% ;(\mathrm{H} / \mathrm{T}) \times 100=5,2 \%$

2 MN: mata natural; MI: cultivo de milho; CL: cultivo da cana, amostragem na linha; CE: cultivo da cana, amostragem na entrelinha; PC: cultivo do cafeeiro, amostragem na projeção da copa; EL: cultivo do cafeeiro, amostragem na entrelinha.

${ }^{3}$ T: C orgânico no solo; AF: C orgânico da fração ácido fúlvico; AH: C orgânico da fração ácido húmico, H: C orgânico da fração humina; MH: C orgânico da fração matérias húmicas. 
(1975) fracionando matéria orgânica de solos ácidos tropicais com $\mathrm{NaOH} 0,1 \mathrm{~N}$, os quais encontraram que, em média, $41 \%$ do $\mathrm{C}$ foi extraído como matéria húmica.

O solo apresentou, em todos os tratamentos, inclusive na mata natural, menores porcentagens de $\mathrm{C}$ orgânico na forma de ácidos fúlvicos do que na forma de ácidos húmicos; isto é um dos fatores que podem colaborar para uma menor lixiviação ou translocação de elementos no perfil do solo (Greenland, 1965).

Por outro lado, predominou no solo o C orgânico na forma de húmus residual (humina), acima de $74 \%$ do $\mathrm{C}$ total, nas duas profundidades (Tabela 2). Este dado é superior ao obtido por Roth et al. (1992), que verificaram valores na faixa de $52,4 \%$ a $63,9 \%$, em um Latossolo Roxo distrófico, em Londrina, PR.

Um alto teor de argilas oxídicas pode ter atuado como fonte de $\mathrm{Al}$ e $\mathrm{Fe}$, complexando e retendo o húmus na forma de humina. Isto confere certa estabilidade na matéria orgânica do solo (Pirakornphanich et al., 1988).

O efeito do manejo do solo e da cultura é evidenciado ao se compararem os teores de $\mathrm{C}$ orgânico das áreas com café com os da área onde foi cultivado por três anos o milho, uma vez que as duas áreas possuem o mesmo histórico até 1991. A área com cultivo do milho apresentou teores de C orgânico iguais ao da área com cana-de-açúcar, apesar de não ter diferido da área com café. Isto indica que três anos de cultivo do milho foram suficientes para apresentar uma tendência de redução no $\mathrm{C}$ orgânico do solo.

Em solo (Entisol) mantido sob gramíneas naturais e cultivado com milho por 40 anos, ocorreram quantidades de C iguais a $36,4 \mathrm{~g} / \mathrm{kg}$ e $10,8 \mathrm{~g} / \mathrm{kg}$, respectivamente (Riffaldi et al., 1994). Isto representa uma redução de até $70 \%$ pelo uso do solo por 40 anos com cultivo do milho, em relação à gramínea natural.

Este fato sugere que ao se alterar o manejo, a matéria orgânica sofre rápidas alterações, atingindo um novo equilíbrio, sendo maior na mata natural, menor em culturas permanentes, e ainda menor nas culturas anuais.

A Tabela 3 indica que as maiores reduções no teor de C orgânico no solo ocorreram na camada de $0-10 \mathrm{~cm}$, e menores, na profundidade de $10-20 \mathrm{~cm}$. A área com cana-de-açúcar apresentou reduções de
$41,3 \%$ a $49,1 \%$ na profundidade de $0-10 \mathrm{~cm}$. Na camada de $10-20 \mathrm{~cm}$, a redução ficou ao redor de $23,7 \%$ a $35,8 \%$.

Cerri (1986) detectou uma redução de 38\% e 46\% no estoque de $\mathrm{C}$ na camada de $0-20 \mathrm{~cm}$ do solo, aos 12 e 50 anos, respectivamente, de cultivo intenso de cana-de-açúcar, valores, estes, próximos aos apresentados na Tabela 3.

A redução do aporte de $\mathrm{C}$ no solo não se deve unicamente à redução da quantidade de resíduos adicionados, mas também, ao aumento da atividade microbiana, causado por melhores condições de aeração, temperaturas mais elevadas e alternância mais freqüente de umedecimento e secagem do solo (Stevenson, 1982), pelo uso contínuo de implementos, pela sucessão contínua, pelas queimadas, e pelas perdas causadas pela própria erosão.

Analisando-se as posições de amostragens (linha e entrelinha), não se observaram diferenças significativas pelo teste de Tukey a 5\% de probabilidade quanto ao teor de $\mathrm{C}$ orgânico do solo e fração humina, na mesma cultura.

A análise estatística dos dados referentes ao $\mathrm{C}$ microbiano do solo detectou efeito significativo a $1 \%$ de probabilidade no uso do solo, e não-significativo quanto à profundidade. Os maiores valores de $\mathrm{C}$ microbiano estavam nos tratamentos com cana-deaçúcar, e os demais tratamentos não diferiram da mata natural (Tabela 4).

O fato de não haver efeito significativo em profundidade pode ser conseqüência de a maior parte da biomassa microbiana se encontrar nos primeiros 0-15 cm superficiais do solo, e de a distribuição desta ser praticamente uniforme (Cerri et al., 1985).

TABELA 3. Porcentagem de redução do $C$ orgânico de um Latossolo Roxo em função de usos agrícolas, em duas camadas.

\begin{tabular}{lcc}
\hline Uso do solo & $0-10 \mathrm{~cm}$ & $10-20 \mathrm{~cm}$ \\
\hline MI & 37,2 & 17,2 \\
CL & 49,1 & 23,7 \\
CE & 41,3 & 35,8 \\
PC & 33,1 & 11,5 \\
EL & 31,9 & 7,9 \\
\hline
\end{tabular}

${ }^{1} \mathrm{MN}$ : mata natural; MI: cultivo de milho; CL: cultivo da cana, amostragem na linha; CE: cultivo da cana, amostragem na entrelinha; PC: cultivo do cafeeiro, amostragem na projeção da copa; EL: cultivo do cafeeiro, amostragem na entrelinha. 
TABELA 4. C microbiano (CM) no solo, em g/kg na terra fina seca em estufa (TFSE), e porcentagem do C microbiano em relação ao C orgânico de um Latossolo Roxo submetido a diferentes usos agrícola, em duas camadas.

\begin{tabular}{|c|c|c|c|c|}
\hline \multirow[t]{2}{*}{ Uso do solo ${ }^{2}$} & \multicolumn{2}{|r|}{$0-10 \mathrm{~cm}$} & \multicolumn{2}{|r|}{$10-20 \mathrm{~cm}$} \\
\hline & C microbiano & $(\mathrm{C}$ microbiano/C orgânico $)$ & $\mathrm{C}$ microbiano & (C microbiano/C orgânico) \\
\hline & (g/kg na TFSE) & x $100(\%)$ & (g/kg na TFSE) & x $100(\%)$ \\
\hline $\mathrm{MN}$ & $0,39 \mathrm{Ba}$ & $1,1 \mathrm{BCa}$ & $0,22 \mathrm{Ba}$ & $0,8 \mathrm{BCa}$ \\
\hline MI & $0,40 \mathrm{ABa}$ & $1,7 \mathrm{BCa}$ & $0,20 \mathrm{Ba}$ & $0,9 \mathrm{BCb}$ \\
\hline CL & $0,66 \mathrm{Aa}$ & 3,5Aa & $0,64 \mathrm{Aa}$ & $3,1 \mathrm{Aa}$ \\
\hline $\mathrm{CE}$ & $0,20 \mathrm{Ba}$ & $0,9 \mathrm{Cb}$ & $0,56 \mathrm{Aa}$ & $3,3 \mathrm{Aa}$ \\
\hline $\mathrm{PC}$ & $0,21 \mathrm{Ba}$ & $0,8 \mathrm{Ca}$ & $0,17 \mathrm{Ba}$ & $0,7 \mathrm{Ca}$ \\
\hline EL & $0,44 \mathrm{ABa}$ & $1,7 \mathrm{Ba}$ & $0,38 \mathrm{ABa}$ & $1,5 \mathrm{Ba}$ \\
\hline
\end{tabular}

1 Médias seguidas da mesma letra, maiúscula nas colunas e minúscula nas linhas, não diferem signifícativamente pelo teste de Tukey a $5 \%$ de probabilidade; coeficientes de variação para tratamentos: (C microbiano $=30,1 \%$ e $(\mathrm{C}$ microbiano/C orgânico $) \times 100=20,7 \%$; coeficientes de variação para profundidades: $\mathrm{C}$ microbiano $=25,9 \%$ e $(\mathrm{C}$ microbiano/C orgânico $) \times 100=12,8 \%$.

2 MN: mata natural; MI: cultivo de milho; CL: cultivo com cana, amostragem na linha; CE: cultivo com cana, amostragem na entrelinha; PC: cultivo do cafeeiro, amostragem na projeção da copa; EL: cultivo do cafeeiro, amostragem na entrelinha

A relação $\mathrm{C}$ microbiano/C orgânico apresentou maior valor nos tratamentos com cana-de-açúcar $(3,1$ a 3,5$)$, exceto para cana (EL) na profundidade de $0-10 \mathrm{~cm}$. Provavelmente, o menor valor encontrado pode ser conseqüência de problemas com a amostragem (Tabela 4). Marchiori Júnior (1998), estudando uma terra roxa estruturada submetida ao cultivo com algodoeiro por dez anos encontrou valores de $\mathrm{C}$ microbiano/C orgânico na faixa de $0,7 \mathrm{a}$ 0,8 . Estes valores estão próximos aos obtidos na mata natural $(1,1$ a 0,8$)$.

O aumento da biomassa microbiana na cana pode ser devido a características intrínsecas à cultura, tais como produtos orgânicos novos gerados pelas plantas cultivadas, principalmente pelas raízes (Warembourg \& Morral, 1978; Sauerbeck et al.,1982).

Com a instalação da agricultura, os teores de C total no solo tenderam a diminuir e não necessariamente levou à redução do $\mathrm{C}$ microbiano do solo. Wardle (1992) cita que os valores de C microbiano nem sempre se relacionam com o $C$ orgânico do solo. $\mathrm{O}$ C orgânico do solo também não se correlacionou com o C microbiano na profundidade de $10-20 \mathrm{~cm} \mathrm{em}$ solo sob mata natural, pastagens e cultivo anual do algodoeiro (Marchiori Júnior, 1998).

\section{CONCLUSÕES}

1. A mata natural apresenta os maiores valores de $\mathrm{C}$ orgânico no solo e na fração humina e os menores valores estão presentes nas áreas com cana-de-açúcar.
2. As áreas com cana-de-açúcar apresentam maiores valores de $\mathrm{C}$ microbiano que a mata natural.

3. Os usos agrícolas do solo aumentam a porcentagem de $\mathrm{C}$ orgânico na forma de ácidos húmicos e fúlvicos.

4. O solo apresenta mais de $74 \%$ do C orgânico na forma de humina.

\section{AGRADECIMENTOS}

À Fundação de Amparo a Pesquisa do Estado de São Paulo (FAPESP), pelo apoio financeiro.

\section{REFERÊNCIAS}

ANDREAUX, F.; BECERRA, S.P. de. Fraccionamiento y caracterización del material húmico en algunos suelos de sabana de la orinoquia Colombiana. Turrialba, San Jose, v.25, n.2, p.1991-1998, 1975.

CERRI, C.C. Dinâmica da matéria orgânica no agrossistema cana-de-açúcar. Piracicaba : USPESALQ, 1986. 197p. Tese de Livre Docência.

CERRI, C.C.; VOLKOFF, B.; EDUARDO, B.P. Efeito do desmatamento sobre a biomassa microbiana em Latossolo Amarelo da Amazônia. Revista Brasileira de Ciência do Solo, Campinas, v.9, n.1, p.1-4, 1985 .

Pesq. agropec. bras., Brasília, v.35, n.6, p.1177-1182, jun. 2000 
DABIN, B. Curso sobre matéria orgânica do solo: análise dos compostos húmicos do solo. Piracicaba CENA, 1976. parte 1 .

DUARTE, P.A. Calagem e sistemas de rotação de culturas: efeitos nas características e propriedades da matéria orgânica do solo. Jaboticabal : UNESP-FCAV, 1994. 165p. Dissertação de Mestrado.

GERALDES,A.P.A.; CERRI, C.C.; FEIGL, B.J. Biomassa microbiana de solo sob pastagens na Amazônia. Revista Brasileira de Ciência do Solo, Campinas, v. 19, p. 55-60, 1995

GREENLAND, D.J. Interaction between clays and organic compounds in soils. 2. Adsorption of soil organic compounds and its effect on soil properties. Soil and Fertilitizers, Wallingford, v.28, p.232-251, 1965 .

INSTITUTO AGRONÔMICO (Campinas, SP). Recomendações de adubação e calagem para o Estado de São Paulo. Campinas, 1996. p.3-6. (IAC. Boletim Técnico, 100)

JENKINSON, D.S.; LADD, J.N. Microbial biomass in soil. Measurement and turnover. In: PAUL, E.A.; LADD, J.N. (Ed.). Soil biochemistry. New York Dekker, 1981. p.415-471.

JENKINSON, D.S.; POWLSON, D.S. The effects of biocidal treatments on metabolism in soil. V. A method for measuring soil biomassa. Soil Biology \& Biochemistry, Elmsford, v.8, p.209-213, 1976.

KLEMEDTSSON, L.; BERG, P.; CLARHOLM, M.; SCHNURER, L. Microbial nitrogen transformation in the root environment of bailey. Soil Biology \& Biochemistry, Elmsford, v.19, p.551-558, 1987.

MARCHIORI JÚNIOR, M. Carbono, nitrogênio e biomassa microbiana e atividade enzimática num solo sob mata natural ou cultivado com pastagem ou algodoeiro. Jaboticabal : UNESP-FCAV, 1998. 70p. Dissertação de Mestrado.

PIMENTEL-GOMES, F. Curso de Estatística Experimental. 3.ed. Piracicaba : USP-ESALQ, 1966. 404p
PIRAKORNPHANICH, P.; WADA, S.I.; WADA, K Metal-humus complexes in a horizon of Thai and Kovan red and yellow soils. Journal of Soil Science, Oxford, v.39, p.529-537, 1988.

POWLSON, D.S.; BROOKES, P.C.; CHRISTENSEN, B.T. Measurement of soil microbial biomass provides an indication of changes in total soil organic matter due to straw incorporation. Soil Biology \& Biochemistry, Elmsford, v.19, n.2, p.159-164, 1987.

RIFFALDI, R.; SAVIOZZI, A.; LEVI-MINZI, R.; MENCHETTI, F. Chemical characteristics of soil after 40 years of continuous maize cultivation. Agriculture, Ecosystems and Environment, Amsterdam, v.49, p.239-245, 1994.

ROTH, C.H.; WILCZYNSKI, W.; CASTRO, F. de C. Effect of tillage and liming on organic matter composition in a Rhodic Ferralsol from Southern Brazil. Zeitschrift fuer Pflanzenernäehrung und Bodenkund, v.155, p.175-179, 1992.

SAUERBECK, D.R.; HELAL, H.M.; NONNEN, S.; ALLARD, J.L. Photosynthate consumption and carbon turnover in the rhizosphere depending on plant species and growth conditions. In: COLOQUIO REGIONAL SOBRE MATÉRIA ORGÂNICA DO SOLO, 1., 1982, Piracicaba. Anais. São Paulo : CENA/PROMOCET, 1982. p.171-174.

STEVENSON, F.J. Humus chemistry, genesis, composition, reaction. New York : J. Wiley, 1982. $443 p$

WARDLE, D.A. A comparative assessment of factors with influence of microbial biomass carbon and nitrogen levels in soil. Biological Review, v.67, p.321-358, 1992.

WAREMBOURG, F.R.; MORRAL, R.A.A. Energy flow in the plant microorganisms system. In: DOMMERGUES, Y.; KRUPA, S.V. (Ed.). Interactions between nonpathogenic soil microorganisms and plants. Amsterdam : Elsevier, 1978. p.205-242. 